\title{
FARINHA DE MARACUJÁ-AMARELO - Passiflora edulis F. Flavicarpa: Aplicabilidade no tratamento da Diabetes Mellitus Tipo II
}

\author{
PASSION FRUIT PEEL FLOUR - Passiflora edulis F. Flavicarpa: Applicability in the treatment \\ of type II Diabetes Mellitus
}

\author{
Aline Luciene Manzoli ${ }^{1}$ \\ Dayelle Lago Araújo ${ }^{2}$ \\ Gabrielle Barreto Julião ${ }^{3}$ \\ Anna Maly de Leão Neves e Eduardo ${ }^{4}$
}

RESUMO: A Diabetes Mellitus tipo II é uma doença consequente de uma série de distúrbios metabólicos, que atualmente afeta a vida de 422 milhões de pessoas no mundo, segundo a Organização Mundial da Saúde. Existem diversas classes de hipoglicemiantes orais usados no tratamento da doença que são altamente eficazes e eficientes. Entretanto, há inúmeros estudos testando a efetividade de tratamentos alternativos e simultâneos, como, a ingestão da farinha de maracujá-amarelo. O objetivo deste trabalho é analisar o perfil glicêmico de diabéticos comparando os mecanismos de ação de hipoglicemiantes orais e a atividade da farinha, a fim de avaliar sua eficácia no tratamento. Trata-se de uma revisão de literatura com base em artigos científicos publicados entre os anos de 2010 e 202I, pesquisados nas bases de dados Scielo, Lilacs, BVS e Pubmed, além de revistas médicas. Perante as considerações apresentadas e avaliadas, conclui-se que a farinha de maracujá-amarelo possui eficácia na redução dos marcadores do perfil glicêmico.

Palavras-chave: Diabetes Mellitus tipo II. Farinha de maracujá-amarelo. Hipoglicemiantes orais. Passiflora edulis F. Flavicarpa.

ABSTRACT: Type II Diabetes Mellitus is a disease resulting from a group of metabolic disorders, which currently affects the lives of 422 million people worldwide, according to the World Health Organization. There are several kinds of oral hypoglycemic agents used to treat the disease that are highly effective and efficient. However, there are uncountable studies testing the effectiveness of alternative and simultaneous treatments, such as the ingestion of passion fruit peel flour. The objective of this paper is to analyze the glycemic

\footnotetext{
'Graduandas do curso de Farmácia, Brasília-DF.

${ }^{2}$ Graduandas do curso de Farmácia, Brasília-DF.

3 Graduandas do curso de Farmácia, Brasília-DF. E-mail: gabriellejbarreto@gmail.com.

4 Professora Orientadora, Brasília-DF.
} 
profile of diabetics by comparing the mechanisms of action of oral hypoglycemic agents and the flour activity, in order to evaluate its effectiveness in the treatment. It is a literature review based on scientific articles published between the years 2010 and 2021, searched in the sources Scielo, Lilacs, BVS, Pubmed, and medical journals. Due of the considerations presented and evaluated, it is concluded that the yellow passion fruit flour is effective in reducing the indicators of the glycemic profile.

Keywords: Oral hypoglycemic agents. Passion fruit peel flour. Passiflora edulis F. Flavicarpa.Type II Diabetes Mellitus.

\section{INTRODUÇÃO}

Diabetes Mellitus tipo II em sua forma mais compreensível, é uma doença onde há uma carência no hormônio insulina. Tecnicamente, pode estar ligada a resistência insulínica ou em decorrência de uma deficiência na produção da insulina (FERREIRA, et al., 2014).

No Brasil, a taxa de diabéticos aponta um aumento de 60\% de pessoas nos últimos io anos. Estimando que 7\% dos brasileiros sofram com a doença (OLIVEIRA, et al., 2018). Segundo a Organização Mundial de Saúde no mundo cerca de 422 milhões de pessoas são diabéticas (WHO, 2020).

Com base na vida que as pessoas levam desde a revolução industrial quando tudo se tornou mais facilitado, e no avanço da tecnologia, onde tudo se consegue com o aperto de um botão, é esperado que a população se torne cada vez mais sedentária e tenha péssimos hábitos alimentares, isso leva à uma estimativa que em 2040 haverá cerca de 642 milhões de pessoas sofrendo de diabetes no mundo, e no Brasil poderá ter um aumento significativo chegando a 26 milhões de pessoas (SANTOS, et al., 2019).

Há uma prática envolvendo o uso de alimentos naturais que simultaneamente entregam algum efeito farmacológico quando em organismo, estes, são denominados alimentos funcionais. Destes, então, é esperado que além dos compostos bioativos resultem em profilaxia, tratamento e/ou cura de doenças específicas (BERNARDES et al., 2010).

O maracujá-amarelo (Passiflora edulis F. Flavicarpa) possui fortes características que o fazem se enquadrar como alimento funcional. Há estudos que mostram a eficácia do fruto no tratamento de pacientes lipêmicos, ajudando a controlar os níveis séricos de colesterol e triglicérides. A Passiflora edulis F. Flavicarpa do gênero Passiflora L pertencente à família 
Passifloraceae é facilmente cultivado no Brasil, devido ao clima tropical e subtropical. As maiores produções do país ocorrem nos estados de São Paulo, Bahia, Sergipe e Minas Gerais (ROBERT, 2018).

O objetivo geral desta revisão foi analisar os efeitos da farinha de maracujá-amarelo no tratamento da diabetes mellitus tipo II, investigando os marcadores do perfil glicêmico quando em uso do alimento. Os objetivos específicos, integraram: descrever o processo de produção da farinha, discorrer sobre a pectina, ressaltar os mecanismos de ação de hipoglicemiantes orais em relação a pectina, apresentar demais benefícios do alimento.

O tema em questão não tem a finalidade de apresentar o alimento como uma alternativa às medicações regulares, e sim mostrar a capacidade terapêutica de um fruto tão regional, acessível e popular, que é o maracujá.

\section{MATERIAL(IS) E MÉTODOS}

Refere-se a um estudo de revisão literária, com estrutura qualitativa e quantitativa, a fim de analisar a aplicabilidade da farinha de maracujá-amarelo no tratamento da diabetes mellitus tipo II.

Verificaram-se artigos publicados entre os anos 2010 e 2021, publicados eletronicamente em revistas científicas, retiradas das bases de dados Scientific Eletronic Library Online (SCIELO), US National Library of Medicine National Institute of Health (PUBMED), Literatura Latino-Americana e do Caribe em Ciências da Saúde (LILACS) e Biblioteca Virtual em Saúde (BVS), em língua inglesa e português brasileiro.

Os critérios de inclusão foram os artigos e publicações relacionados ao conteúdo em proeminência, com os seguintes descritores de busca: diabetes mellitus tipo II farinha de maracujá-amarelo, hipoglicemiantes orais e Passiflora edulis F. Flavicarpa. O presente estudo foi composto por trinta e duas referências, incluindo, artigos de revisão sistemática e bibliográfica, pesquisas clínicas em modelos animais e humanos, sites governamentais e organizacionais. Foram convenientes os artigos presentes nas bases de dados sobre a respectiva temática e foram excluídos periódicos duplicados, incompletos ou não relacionados ao assunto abordado. 


\section{DESENVOLVIMENTO}

\section{I Diabetes mellitus tipo II}

A Diabetes é caracterizada por um conjunto de doenças metabólicas capazes de causar distúrbios na secreção ou ação da insulina. Tecnicamente pode estar ligada a resistência insulínica ou em decorrência de uma deficiência na produção da insulina. Havendo este erro em sua produção, a insulina que é um hormônio anabólico produzidos pelas células $\beta$ (beta) pancreáticas, cuja função é única e relevante no mecanismo de metabolismo de glicose, não mais conseguirá desempenhar o seu papel, consequentemente aumentando os níveis séricos de glicose na corrente sanguínea e não realizando o processo de síntese de ATP (FERREIRA, et al., 2014).

A forma mais comum de diabetes é a Diabetes Mellitus Tipo II (DM), que é de 90 95\% dos casos totais de diabetes. A principal base desta doença é o alto consumo de carboidratos o que consequentemente leva ao metabolismo de glicose pelo organismo. É comum que a maior parte de pacientes com Diabetes mellitus tipo II sofram com problemas de obesidade, ou uma alta porcentagem de gordura distribuída principalmente na região abdominal, resultado do sedentarismo (DIABETES CARE, 2013).

O diagnóstico de DM é através de um padrão de marcadores bioquímicos essenciais para avaliação dos níveis de glicose no sangue. Os principais deles são: Glicose plasmática em jejum de $8 \mathrm{~h}(\mathrm{FPG})(\geq \mathrm{I} 26 \mathrm{mg} / \mathrm{dl})$, glicemia 2 horas pós-prandial ou TOTG após a administração oral de $75 \mathrm{mg}$ de dextrose $(\geq 200 \mathrm{mg} / \mathrm{dl})$ e hemoglobina glicada (HbAIc) ( $\geq$ 6.5\%)que é capaz de mensurar os níveis de glicose por um período de 2 a 3 meses (DIABETES CARE, 2013).

\section{I.I Diabetes Mellitus Tipo II: Tratamento}

O atual tratamento da DM tipo II é feito principalmente através de hipoglicemiantes orais, estes são divididos em classes, e a escolha é feita através de uma melhor assimilação para a necessidade do diabético. São elas: 
Tabela I - Classes de hipoglicemiantes orais e seus respectivos mecanismos de ação, alguns de seus fármacos e efeitos hipoglicemiantes.

\begin{tabular}{|c|c|c|c|c|}
\hline CLASSE & FARMACODINÂMICA & FÁRMACOS & HBAiC & $\begin{array}{l}\text { GLICEMIA } \\
\text { JEJUM }\end{array}$ \\
\hline Sulfoniluréias & $\begin{array}{l}\text { Atuam nas células } \beta \text { pancreáticas } \\
\text { estimulando a secreção de insulina } \\
\text { pelo pâncreas, através do bloqueio } \\
\text { de canais de potássio dependentes de } \\
\text { adenosina trifosfato (K+\ATP) da } \\
\text { subunidade I do receptor de } \\
\text { sulfoniluréia, ocorre então a } \\
\text { despolarização da membrana, o } \\
\text { influxo de Ca+ e a liberação de } \\
\text { insulina. }\end{array}$ & $\begin{array}{l}\text { Glipizida } \\
\text { Glicazida } \\
\text { Glimepirida }\end{array}$ & ++ & + \\
\hline Biguanidas & $\begin{array}{l}\text { Funcionam através da ativação da } \\
\text { proteína quinase dependente de } \\
\text { adenosina monofosfato (AMPK) } \\
\text { nas células hepáticas, bloqueiam e } \\
\text { degradam os ácidos graxos, dessa } \\
\text { forma a uma inibição da } \\
\text { gliconeogênese e da glicogenólise } \\
\text { hepática. Consequentemente é } \\
\text { esperado a redução da resistência } \\
\text { insulínica. }\end{array}$ & Metformina & ++ & ++ \\
\hline $\begin{array}{l}\text { Inibidores da } \alpha- \\
\text { glicosidase }\end{array}$ & $\begin{array}{l}\text { Agem poupando a insulina antes das } \\
\text { refeições, liberando GLP-I, que } \\
\text { possuem capacidade de inibir a } \\
\text { liberação do polipeptídeo inibidor } \\
\text { gástrico (GIP). }\end{array}$ & Acarbose & ++ & + \\
\hline $\begin{array}{c}\text { Glitazonas ou } \\
\text { Tiazolidinedionas }\end{array}$ & $\begin{array}{l}\text { Ativam o fator de transcrição } \\
\text { nuclear PPARy, que possui } \\
\text { capacidade de alterar a transcrição } \\
\text { de genes, modificando as proteínas. } \\
\text { Alteram o ambiente metabólico de } \\
\text { hepatócitos e miócitos, levando a } \\
\text { um aumento de sensibilidade à } \\
\text { insulina, por estes. }\end{array}$ & Pioglitazona & ++ & + \\
\hline $\begin{array}{c}\text { Inibidores de } \\
\text { Dipeptidil-peptidase- } \\
\text { IV }\end{array}$ & $\begin{array}{l}\text { Atuam inibindo a enzima DPP-IV, } \\
\text { que são capazes de degradar os } \\
\text { hormônios GLP I que estimula a } \\
\text { secreção de insulinas das células } \\
\text { beta pancreáticas limitando a } \\
\text { liberação de glucagon pelo pâncreas. } \\
\text { Inibir a enzima que o degrada } \\
\text { aumenta a concentração sanguínea e } \\
\text { é esperado então a secreção de } \\
\text { insulina. }\end{array}$ & $\begin{array}{l}\text { Sitagliptina } \\
\text { Vildagliptina } \\
\text { Saxagliptina }\end{array}$ & + & + \\
\hline Inibidores de SGLT2 2 & $\begin{array}{l}\text { Reduzem a hiperglicemia através do } \\
\text { processo de bloquear a reabsorção } \\
\text { renal de glicose, dessa forma a } \\
\text { excreção de urina é aumentada. Ou }\end{array}$ & $\begin{array}{l}\text { Canagliflozina } \\
\text { Dapagliflozina } \\
\text { Empagliflozina } \\
\text { Ertugliflozina }\end{array}$ & +++ & +++ \\
\hline
\end{tabular}


(+): resultado regular; $(++)$ : resultado bom; $(+++)$ : resultado significativo.

Fonte: Elaborado pelas autoras com base em DUARTE, 2013; SOCIEDADE BRASILEIRA DE DIABETES, 2018 e PIOLI et al., 2017.

\subsection{Maracujá-Amarelo}

O maracujá tem sua origem na América tropical, e é produzido pelas plantas do gênero Passiflora, onde mais de 150 espécies são utilizadas para o consumo humano (EMBRAPA, 2016). As três principais espécies produzidas no Brasil e no mundo são a do maracujá-amarelo (Passiflora edulis F Flavicarpa - PEFF), maracujá-doce (Passiflora alata) e maracujá roxo (Passiflora edulis Sims). O do tipo amarelo é considerado o mais cultivado mundialmente (FAVERI, 2019).

O maracujá-amarelo ou maracujá azedo tem seu fruto do tipo baga, de forma subglobuloso ou oval. Seu tamanho e coloração podem ter várias variações, tendo em média de $7 \mathrm{~cm}$ de comprimento por $6 \mathrm{~cm}$ de largura, e seu peso pode variar de 44 e r6og. Sua casca é coriácea e a cor amarelo intenso, ocorrendo no fim do amadurecimento (FAVERI, 2019).

A região Sudeste é a que possui maior produção e consumo de pomares de maracujá no Brasil. A melhor época para o cultivo da planta é entre o final de agosto e o final de novembro, durando aproximadamente 6 meses por ano. Na região Norte o tempo de cultivo poderá perdurar um ano inteiro. Alguns fatores afetam cotidianamente a produtividade, como condições climáticas ou os tratos culturais, com isso dificulta a longevidade da produção, limitando-a num período de até dois anos (EMBRAPA, 2018).

\subsection{Propriedades nutricionais e potencial terapêutico}

Análises químicas do Passiflora edulis F. Flavicarpa (PEFF) apresentaram teores excepcionais de propriedades nutricionais na casca (Ioog) do fruto (ZERAIK et al., 20Io):

- Umidade $(87,64 \mathrm{~g})$

- Cinzas $(0,57 g)$

- Lipídeos (o,org) 
- Proteínas (o,67g)

- Fibras $(4,33 \mathrm{~g})$

- Carboidratos $(6,78 \mathrm{~g})$

- Calorias (29,9rkcal)

- Cálcio (44,5img)

- Ferro (o,89mg)

- Sódio $(43,77 \mathrm{mg})$

- Magnésio (27,82mg)

- Zinco (o,32mg)

- Cobre (o,04mg)

- Potássio (178,40mg)

Sua produção pode ajudar na medicina popular para o tratamento de algumas enfermidades como: atividade antioxidante, atividade anti-hipertensiva, redução em níveis séricos de colesterol, HDL e glicose, tratamento da insônia, ansiedade e irritabilidade. As folhas também podem ser usadas para combater inflamações cutâneas, erisipela e febres intermitentes (PITA, 2012). Além de serem frutos ricos em alcaloides, flavonoides, carotenoides, minerais e vitamina A e C (ZERAIK et al., 2010).

A farinha de maracujá contém potencial antioxidante, antimicrobiano e também pode atuar na prevenção de doenças cardiovasculares e Alzheimer, auxiliando na redução dos radicais livres, pois contém os polifenóis. Os polifenóis são importantes na prevenção das doenças cardiovasculares, capaz de promover o aumento das defesas oxidativas, e auxiliam também os níveis de acetilcolina no cérebro, fazendo que a ação da enzima acetilcolinesterase (AChE) tenha uma redução, esta é envolvida na doença de Alzheimer (LIMA et al., 2018).

O maracujá é usado na alimentação humana de várias formas, onde suas principais utilizações podem ser: sucos, geleias, refrescos, sorvetes e doces. A polpa e o suco são utilizados no preparo de vários produtos como xaropes, bebidas mistas, sucos em pó, laticínios entre outros. A parte mais consumida é o fruto, já a casca do maracujá pode ser utilizada para a fabricação de farinha. As sementes e cascas correspondem de 65 a $70 \%$ do peso do fruto (SANTOS, 2017). 
3.3 Farinha de maracujá-amarelo - Passiflora Edulis F. Flavicarpa (FPEFF): Produção, comercialização e aquisição

A farinha do maracujá-amarelo (FPEFF) é hoje classificada como um alimento funcional pois possuem diversas atividades farmacológicas além das funções nutricionais, sendo assim, o seu consumo pode levar a prevenção ao mesmo um auxílio no tratamento de algumas doenças (ZERAIK, et al., 20I0).

Após os processos de seleção, sanitização, despolpa e descasca é então obtido somente o mesocarpo. Na produção da farinha de maracujá o mesocarpo é utilizado após o processo de desidratação em estufa, este processo pode levar até is horas para ser concluído. Para a enfim obtenção da farinha o mesocarpo desidratado é submetido à trituração em um liquidificador e peneirado (LIMA et al., 2012).

Figura I - Processo de produção

\section{OBTENÇÃO DA FARINHA DE MARACUJÁ-AMARELO}

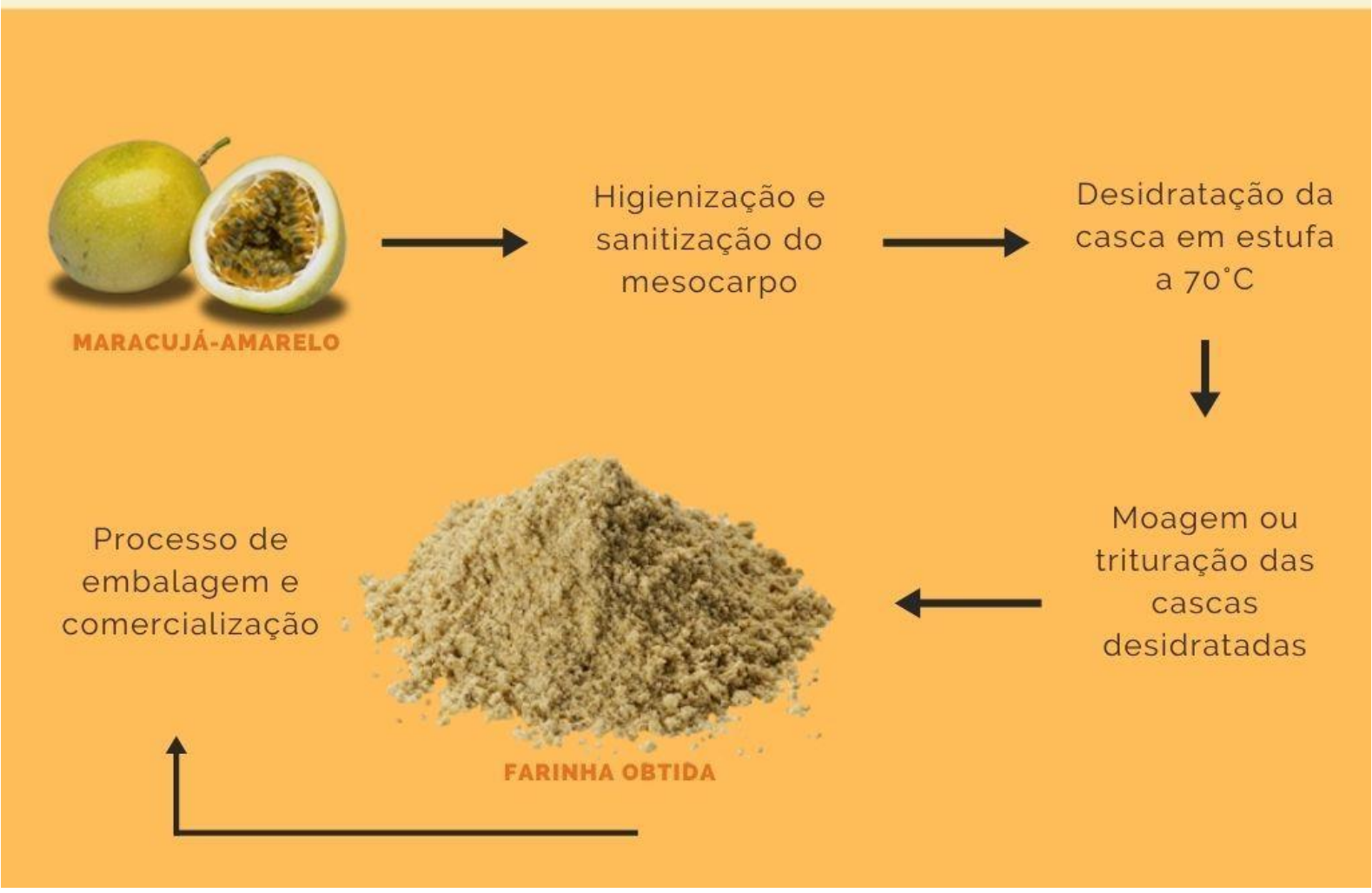


Fonte: Elaborado pelas autoras

A comercialização da FPEFF começou após as divulgações positivas sobre seus efeitos benéficos ao organismo. A partir de 2003, artesanalmente inúmeras pessoas passaram a fabricar sua própria farinha com intuito de emagrecer e reduzir os níveis glicêmicos, principalmente. Não demorou muito para que indústrias e grandes empresas começassem a fabricar em larga escala e comercializar a farinha, de forma terapêutica (BRAGA et al., 20ro).

Segundo pesquisa de preço realizada pelas autoras, a FPEFF pode ser facilmente encontrada em feiras, casas de produtos naturais e lojas online, com o custo de $\mathrm{R} \$ 12,52$ a $\mathrm{R} \$ 24,90$ a embalagem com $\mathrm{ikg}$.

\subsection{Toxicidade da casca do maracujá}

Alguns produtos à base de plantas podem ser considerados seguros, porém sua toxicidade não pode ser descartada devido aos constituintes cianogênios de várias espécies, sendo entre elas as Passifloras (CLARO et al., 2018). As plantas que contém a substância ácido cianídrico (HCN), são consideradas cianogênicas (NASCIMENTO et al., 2013). O HCN é encontrado ligado aos carboidratos onde é chamado de glicosídeos cianogênicos, podendo ser liberado após sua hidrólise. Os glicosídeos cianogênios são substâncias encontradas nas cascas dos maracujás, essa substância age como defesa dos vegetais contra seus predadores (COQUEIRO et al., 2016).

A presença de glicosídeos cianogênicos tem sido estudado principalmente em aspectos toxicológicos do maracujá. $\mathrm{Na}$ ação das enzimas ( $\beta$-glucosidases) ocorre a cianogênese, estas são presentes em outros compartimentos celulares das plantas (DEUS et al., 2014). No entanto, ocorre a cianogênese quando a compartimentalização é rompida, o substrato e a enzima entram em contato, ocorrendo uma defesa química. O cianeto libera íons de cianeto na circulação sanguínea, sendo transportado pela hemoglobina. O cianeto liga-se ao citocromo mitocondrial, sendo responsável pelo transporte de elétrons na respiração celular, podendo então o intoxicado passar por um processo de asfixia celular, chegando ao óbito dependendo da quantidade de cianeto na corrente sanguínea (CLARO et al., 2018). 


\subsection{Pectina: atividade hipoglicemiante}

Uma propriedade contida especialmente no mesocarpo do maracujá- amarelo é a pectina, que é uma fibra solúvel e polissacarídica. A pectina quando fermentada no intestino grosso consegue sintetizar ácidos graxos de cadeia curta, como, acetato, butirato e propionato. O butirato foi diversamente estudado e já é comprovado a sua associação à redução da resistência insulínica, consequentemente reduzindo os níveis séricos de glicose no sangue. Já o propionato após ser metabolizado no fígado consegue estimular a gliconeogênese e a síntese de lipídeos. A pectina corresponderá a 19,1\% da composição do maracujá-amarelo (BRAGA et al.,2010).

Polissacarídeos de alta massa molecular presentes na parede celular de todas as plantas. As pectinas unem as paredes celulares. São usadas como emulsificantes e estabilizantes na indústria alimentícia. Têm sido testadas para uma variedade de usos terapêuticos, inclusive como agentes antidiarreicos (em que geralmente não são mais consideradas efetivas), e no tratamento da hipercolesterolemia (DECS., 2020).

O mesocarpo do maracujá-amarelo é rico em pectina, esta substância possui a capacidade de formar uma camada gelatinosa que consegue modificar a absorção de nutrientes e carboidratos, dessa forma consegue realizar ação hipoglicemiante. Assim então, esse composto se mostra eficaz no tratamento da diabetes, como componente adjuvante (LIMA et al., 2012)

Além da atividade hipoglicemiante a FPEFF é muito procurada por suas propriedades no combate à redução de colesterol, que reduz significativamente os níveis de colesterol total e colesterol LDL, tornando o produto um alimento funcional seguro no combate a hipercolesterolemia (GROSSELI et al., 2014).

Para a obtenção da pectina o processo contém três estágios: Primeiramente é a da extração aquosa do material da planta, a segunda purificação do extrato líquido e o terceiro separação do extrato da pectina do líquido (SILVA, 2015).

A pectina na indústria alimentícia e farmacêutica possui várias aplicações, sendo usado na indústria no aumento da viscosidade dos alimentos, em preparações de bebidas e sucos, produção de geleias e doces entre outros (LIMA, 2019). 
As características da matéria-prima do maracujá são importantes para a obtenção de uma pectina de boa qualidade, reduzindo gastos operacionais de pré-tratamento da matériaprima e garantindo maior rendimento na produção. A pectina é obtida de cascas de frutas, são eliminadas muitas vezes como resíduos pelas indústrias, com isso podem ser susceptíveis a ação de microrganismos, prejudicando a obtenção do produto final (NASCIMENTO et al., 2013). A etapa de armazenamento da matéria-prima é importante para a garantia de um produto de alta qualidade (LIMA, 2019).

A pectina através da suplementação da farinha de maracujá-amarelo também mostrou eficácia no tratamento de síndrome metabólica, após um estudo feito com pacientes diabéticos. A terapia do alimento funcional deve ser feita de forma complementar aos hipoglicemiantes tradicionais (JANEBRO et al., 2010).

\subsection{Alimentos funcionais}

Em meados dos anos 8o no Japão, surgiu uma terapia onde envolvia o uso de alimentos funcionais, as indústrias começaram a produzir alimentos com ingredientes específicos que desempenhavam algum efeito farmacológico quando em organismo $\mathrm{A}$ escassez de uma legislação que padronizasse essa terapia mundialmente oriundo de várias termos nominais, como, nutracêutico, alimentos medicinais e "farma-alimentos" (BERNARDES, 2010).

No Brasil, a Agência Nacional de Vigilância Sanitária (ANVISA) dispôs o conceito e utilização precisa e racional de alimentos funcionais através de uma Comissão de Assessoramento Técnico Científico em Alimentos Funcionais e Novos Alimentos (CTCAF) que foi criada com objetivo de estabelecer parâmetros de comercialização e normas para este tipo de alimento. Esta comissão conceitua para alegação de alimentos com propriedade funciona, o seguinte:

\footnotetext{
É aquela relativa ao papel metabólico ou fisiológico que o nutriente ou não nutriente tem no crescimento, desenvolvimento, manutenção e outras funções normais do organismo humano (ANVISA, 1999)
}

Um alimento deve possuir benefícios nutricionais à saúde e propriedades básicas para prevenção de doenças, para ser considerado como alimento funcional. Entre os alimentos 
definidos como funcionais inclui-se a farinha de maracujá, produzida feita a partir do mesocarpo do fruto, este, possui elevado teor de fibras solúveis, como a pectina (CASTRO, 2018).

\subsection{Atividade hipoglicemiante da farinha de maracujá}

$\mathrm{Na}$ tentativa de encontrar produtos naturais que conseguem tratar ou mesmo auxiliar o tratamento, ao longo dos últimos anos foram feitos alguns testes com FPEFF.

Um estudo realizado com 43 voluntários diabéticos do tipo DM II, ingeriram farinha de maracujá-amarelo por 60 dias. Dos pacientes voluntários em tratamento com hipoglicemiantes, 9 faziam já o uso de gliburida, 9 faziam o uso de metformina, II associavam gliburida com metformina, 7 usavam metformina e insulina, I usava gliburida $e$ insulina, 5 usavam insulina e outro I apenas não estava sendo tratado com hipoglicemiantes orais. Os exames bioquímicos realizados foram: Glicemia, Hemoglobina Glicada (HbAic), Insulina, HOMA IR e HOMA Beta. As testagens foram feitas antes do início do consumo de farinha de maracujá-amarelo (To), 30 dias após a ingestão diária ( após (T6o) (QUEIROZ, de et al., 2012).

Ratos machos da linhagem Wistar foram induzidos a diabetes mellitus através da administração de aloxano monoidratado por via lateral da cauda. Os animais receberam o tratamento da casca do maracujá em diferentes doses. Anteriormente, divididos em 3 grupos: Grupo I - diabético tratado com dose de $20 \mathrm{mg} / \mathrm{Kg}$, Grupo II -diabético tratado com dose de $40 \mathrm{mg} / \mathrm{Kg}$ e Grupo III - diabético tratado com a dose de $160 \mathrm{mg} / \mathrm{Kg}$. A glicemia após o tratamento foi mensurada através de fitas reativas e glicosímetro Prestige IQ, a coleta e dosagem foram realizadas nos tempos o,I, 2 e 4 horas. $\mathrm{Na}$ primeira dosagem dos níveis glicêmicos os animais apresentaram uma média de 525,16 mg/dL (BRAGA et al., 2010).

Uma pesquisa sobre a eficácia de aveia e da farinha de maracujá sobre parâmetros bioquímicos como: glicose, colesterol total, colesterol HDL e triglicérides foi realizada com 28 voluntários sedentários que possuíam uma propensão a desenvolverem síndrome metabólica, estes indivíduos foram divididos em 2 grupos: Grupo I - Aveia e Grupo 2 Farinha de maracujá. Do total de voluntários i6 fizeram parte do Grupo 2, estes receberam 
pacotes contendo $30 \mathrm{~g}$ de farinha de maracujá, suficiente para o tempo do estudo. As coletas e análises foram realizadas em jejum de I2 horas, em tempo basal (To), 30 dias após ( 6o dias após (T6o) (MIRANDA et al., 2014).

Tabela 2 - Resultado dos estudos supracitados.

\begin{tabular}{|c|c|c|c|c|}
\hline \multicolumn{4}{|c|}{ ESTUDO } & REFERÊNCIA \\
\hline \multicolumn{5}{|l|}{ Estudo-I } \\
\hline \multirow[t]{2}{*}{ Parâmetro } & Tempo & \multicolumn{2}{|c|}{ Média } & \multirow{7}{*}{ Queiroz et al., 2012} \\
\hline & To & 162 & $\mathrm{dL}$ & \\
\hline \multirow[t]{3}{*}{ Glicemia } & $\mathrm{T}_{30}$ & 138, & $/ \mathrm{dL}$ & \\
\hline & & & & \\
\hline & T6o & 120 & dL & \\
\hline \multirow[b]{2}{*}{ HbAic } & To & \multicolumn{2}{|c|}{$6,58 \%$} & \\
\hline & T6o & \multicolumn{2}{|c|}{$5,71 \%$} & \\
\hline \multicolumn{4}{|l|}{ Estudo-2 } & \multirow{7}{*}{ Braga et al., 2010 } \\
\hline & & cemia em mg/ & & \\
\hline Tempo & Grupo I & Grupo II & Grupo III & \\
\hline o & $558,5 \mathrm{mg} / \mathrm{dL}$ & 498,o mg/Dl & $493,6 \mathrm{mg} / \mathrm{dL}$ & \\
\hline Ih & $524,5 \mathrm{mg} / \mathrm{dL}$ & $428,5 \mathrm{mg} / \mathrm{dL}$ & $418,2 \mathrm{mg} / \mathrm{dL}$ & \\
\hline $2 \mathrm{~h}$ & $454,3 \mathrm{mg} / \mathrm{dl}$ & $4 \mathrm{I} 3,8 \mathrm{mg} / \mathrm{dL}$ & $315,4 \mathrm{mg} / \mathrm{dL}$ & \\
\hline $4 \mathrm{~h}$ & $390,1 \mathrm{mg} / \mathrm{dL}$ & $337, \mathrm{I} \mathrm{mg} / \mathrm{dL}$ & $237,7 \mathrm{mg} / \mathrm{dL}$ & \\
\hline \multicolumn{5}{|l|}{ Estudo-3 } \\
\hline Tempo & \multicolumn{3}{|c|}{ Glicemia em mg/Dl } & \\
\hline To & \multicolumn{3}{|c|}{ 91,0o mg/Dl } & \\
\hline $\mathrm{T}_{30}$ & \multicolumn{3}{|c|}{$88,43 \mathrm{mg} / \mathrm{Dl}$} & Miranda et al., 2014 \\
\hline T6o & \multicolumn{3}{|c|}{ 8o,oo mg/Dl } & \\
\hline
\end{tabular}

Fonte: Elaborado pelas autoras com base em QUEIROZ et al., 2012; BRAGA et al., 2010 e MIRANDA et al., 2014.

Ratos sofreram a indução de diabetes através de uma injeção de $150 \mathrm{mg} / \mathrm{kg}$ de aloxano monoidratado. Os animais foram divididos em Grupo I recebeu apenas água (1o $\mathrm{mL} / \mathrm{Kg}$ ), o Grupo II recebeu $20 \%$ da farinha, desta vez, farinha de maracujá-do-mato, incorporada na 
ração comercial, e o Grupo III recebeu 40\% da farinha também presente na ração. Os níveis de glicemia basal em três dias após a aplicação foi de $>250 \mathrm{mg} / \mathrm{dL}$. Os animais se mantiveram na dieta por 2I dias. Após a introdução da farinha da dieta, as amostras de sangue foram determinadas através de um glicosímetro ACCU-CHEK ${ }^{\circledR}$ em um intervalo de 1,3,6,9,12,15,18 e 2I dias. Pela primeira vez foi comprovada a eficácia da farinha de maracujá-do-mato (Passiflora nitida Kunth) na redução da glicemia em ratos diabéticos (LIMA et al., 2012).

Um estudo alternativo teve como objetivo produzir uma massa tipo talharim a partir da farinha de casca de maracujá, e consequentemente avaliar a resposta nos níveis séricos de glicose. A massa de talharim teve como ingredientes ovo, farinha de trigo sem fermento, azeite e a farinha da casca de maracujá, em concentrações de $25 \%$ e $45 \%$ relacionada a farinha de trigo. Os 20 voluntários participantes do estudo tiveram a primeira coleta em jejum de I2 horas, a amostra foi coletada por uma punção capilar e analisada em uma fita reagente para glicosímetro ACCU-CHEK ACTIVE ${ }^{\circledR}$. Posteriormente os voluntários ingeriram uma porção de $50 \mathrm{~g}$ da massa de talharim controle, e foram novamente submetidos às coletas nos tempos 30, 6o e 120 minutos. Após 30 dias os mesmos voluntários participaram do mesmo procedimento, porém, com a ingestão da massa de talharim contendo a FPEFF. Os resultados alcançados mostraram a redução do nível sérico de glicose (COSTA et al., 2015).

Uma investigação sobre a sensibilidade insulínica foi avaliada em um estudo cujo objetivo era avaliar os parâmetros metabólicos de ratos jovens com resistência insulínica induzida por frutose $10 \%$ por oito semanas. Além de resistência insulínica os animais adquiriram depósitos de gordura no fígado e aumento dos níveis séricos de triglicérides. $\mathrm{O}$ grupo de ratos que receberam a suplementação com a FPEFF não adquiriram as irregularidades, isto, devido aos compostos bioativos presentes. Os resultados foram identificados por métodos analíticos e são inéditos por apresentar a capacidade da FPEFF de prevenção de esteatose hepática induzida nos roedores e resistência à insulina (GOSS, et al., 2018).

De fato, até mesmo em diferentes dosagens, a suplementação com a FPEFF se mostra eficiente na redução dos níveis séricos de glicemia, colesterol total, triglicérides, LDL e valores da pressão arterial. Além disso, foi comprovada sua eficácia na redução de massa 
corporal em modelos animais. Tudo isto se deve as atribuições do FPEFF enquanto alimento funcional, estas respostas fisiológicas são atribuídas diretamente à pectina, cuja ação terapêutica ocorre através da sua atividade de gelificação, capaz de diminuir a absorção de lipídeos e carboidratos, reduzir níveis séricos glicêmicos e promover a saciedade devido ao aumento do bolo alimentar e viscosidade no TGI (CLARO, et al., 2018).

\section{CONCLUSÃO}

Conclui-se que neste presente estudo além dos muitos benefícios da farinha de maracujá, esta, possui boa aplicabilidade no perfil glicêmico, ou seja, demonstra um efeito positivo na redução dos níveis séricos de glicose em pacientes portadores de Diabetes Mellitus tipo II. Sendo um produto acessível, popular e regional para ser utilizado na terapia nutricional e farmacológica do DM. Sua ação pode ser percebida logo nos primeiros meses de uso, onde a ação hipoglicemiante da farinha de casa de maracujá amarelo é proveniente da presença da pectina, uma fibra solúvel que auxilia na redução da absorção da glicose no organismo.

Entretanto, um profissional de saúde deve sempre ter cautela ao recomendar o consumo da FPEFF por pacientes diabéticos, deverá salientar sempre a importância de não interromper o tratamento terapêutico convencional adotado, pois o alimento funcional não substitui a necessidade do tratamento farmacológico e apenas deverá ser utilizado de forma simultânea.

Por fim, recomenda-se que sejam desenvolvidos mais estudos experimentais e clínicos para avaliar o potencial de toxicidade da farinha de maracujá-amarelo, para que se estabeleça a dosagem adequada a ser consumida e o limite de segurança, a fim de alcançar maior eficácia e benefício na saúde da população.

\section{REFERÊNCIAS}

AMERICAN DIABETES ASSOCIATION. Diagnosis and Classification of Diabetes Mellitus. Diabetes Care, vol. 36, n⿳⼝ㅗ, p. 67-74, 2013.

\section{ANVISA, Agência Nacional de Vigilância Sanitária. Resolução n 18, de 30 de abril de 1999}

- Português (Brasil). Disponível em: <https://www.gov.br/agricultura/pt- 
$\mathrm{br} /$ assuntos/inspecao/produtos-vegetal/legislacao-I/biblioteca-de-normas-vinhos-ebebidas/resolucao-no-I8-de-30-de-abril-de-1999.pdf/view>. Acessado em: 30 abr. 202I.

BERNARDES, Natália Ribeiro. Functional Foods: A Brief Review. Revista Científica Multidisciplinar do Centro Universitário da Fundação Educacional de Barretos, vol. 6, nํㅡ, p. II-78, 2010.

BRAGA, Andressa, et al. Investigação da atividade antihiperglicemiante da farinha da casca de Passiflora edulis sims passifloraceae, em ratos diabéticos induzidos por aloxano. Revista Brasileira de Farmacognosia, vol. 20, n² 2, p. I86-91, 2010.

CASTRO, Jéssica Silva. O uso da farinha de maracujá associado à redução da glicemia em portadores de diabetes mellitus tipo II. Faculdade Atenas Paracatu-MG, 2018.

CLARO, Maísa de Lima, et al. Propriedades funcionais da casca do maracujá amarelo (Passiflora edulis) na síndrome metabólica. DEMETRA: Alimentação, Nutrição \& Saúde, vol. 13, no $1,2018$.

COQUEIRO, A.Y, et al. Farinha da casca do fruto de Passiflora edulis F. Flavicarpa Deg (maracujá-amarelo): do potencial terapêutico aos efeitos adversos. Revista Brasileira de Plantas Medicinais, vol. i8, no 2, p. 563-569, 2016.

COSTA, Elizabete Lourenço da, et al. Produção e aceitação de massa fresca tipo talharim enriquecida com farinha de maracujá (Passiflora edulis sims F. Flavicarpa) e verificação do seu efeito na glicemia. Nutrire, vol. 40, $\mathrm{n}^{\circ} 3, \mathrm{p} .352-60,2015$.

DeCs, Descritores em Ciência da Saúde. Pectinas. Disponível em: 〈http://decs2or6.bvsalud.org/cgi-bin/wxisi66o.exe/decsserver/Pectinas〉. Acessado em: 4 de abril de 202I.

DEUS, Gilciléia. Efeitos da temperatura de secagem nos teores de compostos cianogênicos totais da casca de maracujá. Revista de Alimentação Humana, vol. 20, no 2, p. 68-74, 2014.

DUARTE, Rui. Antidiabéticos orais. Como começar e combinar sem risco cardiovascular. Revista Portuguesa de Cardiologia, vol. 32, p. 15-24, 2013.

EMBRAPA, Empresa Brasileira de Pesquisa Agropecuária. Maracujá: O produtor pergunta, a Embrapa responde. Disponível em: 〈https://mais5oop5oor.sct.embrapa.br/view/publicacao.php?publicacaoid=90000036> Acessado em: 4 de abril de 2021. 
FAVERI, Aline. Efeitos da farinha da casca de maracujá (Passiflora edulis var. flavicarpa) sobre a síndrome metabólica induzida por dieta de cafeteria em camundongos. Universidade do Vale do Itajaí, p, I-II2, 2019.

FERREIRA, Liliane Martins. Physical assessment, and physical chemistry and estimates of genetic parameters 32 genotypes passionfruit sour cultivated in Federal District. Bioscience Journal, vol. 30, p. 36o-370, 2014.

GOSS, M.J., et al. Peel flour of Passiflora edulis Var. Flavicarpa supplementation prevents the insulin resistance and hepatic steatosis induced by low-fructose-diet in young rats. Biomedicine \& Pharmacotherapy, vol. 102, p. 848-854, 2018.

GROSSELI, Marcela. Uso da polpa e da casca do maracujá (Passiflora edulis F. Flavicarpa) sobre o colesterol em coelhos com hipercolesterolemia experimental. Revista de Pesquisa e Inovação Farmacêutica, vol. 6, p. 12-20, 2014.

JANEBRO, D. I., et al. Análise dos componentes da síndrome metabólica antes e após a suplementação dietética com farinha da casca do maracujá, em pacientes diabéticos. Revista brasileira de análises clínicas, vol. 42, p. 217-22, 2010.

LIMA, Denise Simim, et al. Passion fruit and apple: from residues to antioxidant, antimicrobial and anti-Alzheimer's potential. Ciência Rural, vol. 48, no 9, 2018.

LIMA, E. S., et al. Efeito hipoglicemiante da farinha do fruto de maracujá-do-mato (Passiflora nitida kunth) em ratos normais e diabéticos. Revista Brasileira de Plantas Medicinais, vol. $14, \mathrm{n}^{\circ}$ 2, p. 383-88, 2012.

LIMA, Rodrigo. Extração da pectina do maracujá amarelo (Passiflora edulis F. Flavicarpa) para incorporação em biofilmes. Universidade Tecnológica Federal do Paraná, p. I-46, 2019.

MIRANDA, Silva Gilson. Efeito do consumo da aveia e farinha da casca de maracujá sobre a glicemia e lipemia em um grupo de voluntários Revista de Ciências Farmacêuticas Básica e Aplicada, vol. 35, p. 245-50, 2014.

NASCIMENTO, Elisabete Maria da Graça Costa, et al. Benefícios e perigos do aproveitamento da casca de maracujá (Passiflora edulis) como ingrediente na produção de alimentos. Revista do Instituto Adolfo Lutz, vol. 72, no I, p. I-9, 2013.

PIOLI, Mariana. Effects of oral antidiabetics on blood pressure. International Research Journal of Diabetes and Metabolism, vol. 23, p. 90-98, 2017. 
PITA, Julyane. Caracterização físico-química e nutricional da polpa e farinha da casca de maracujazeiros do mato e amarelo. Universidade Estadual do Sudoeste da Bahia, p. I-77, 2012.

QUEIROZ, Maria do Socorro Ramos, et al. Effect of the Yellow Passion Fruit Peel Flour (Passiflora Edulis F. Flavicarpa) in Insulin Sensitivity in Type 2 Diabetes Mellitus Patients. Nutrition Journal, vol. II, nº 1, p. 89, 2012.

ROBERT, Carlison. Nutracêuticos na prática clínica: performance e saúde. Nutropharm Body and Mind, p. $1-57,2018$.

SANTOS, Francisca. História, uso local e caracterização física e química do maracujáamarelo (Passiflora edulis F. Flavicarpa O. Deg.). Universidade Federal de Campina Grande, p.I-75, 2017.

SANTOS, Ranailla Lima Bandeira. Fatores associados à qualidade de vida de brasileiros e de diabéticos: evidências de um inquérito de base populacional. Ciência \& Saúde Coletiva, vol. 24, n. 3, p. 1007-1020, 2019.

SBD, Sociedade Brasileira de Diabetes. Diretrizes 2017-2018. Clannad, disponível em: 〈https://www.diabetes.org.br/profissionais/images/2017/diretrizes/diretrizes-sbd-20172018.pdf $>2017$ Acessado em 3 de abril de 2021.

SILVA, Neiton. Desidratação De Resíduos Do Processamento De Maracujá-Amarelo Por

Diferentes Metodologias. Universidade Federal de Uberlândia, p. I-164, 2015.

WHO, World Health Organization. Diabetes. Disponível em: $\langle$ https://www.who.int/news-room/fact-sheets/detail/diabetes $\rangle$. Acessado 3 de abril de 2021.

ZERAIK, Maria Luiza, et al. Maracujá: Um Alimento Funcional? Revista Brasileira de Farmacognosia, vol. 20, nº 3 , p. 459-71, 2010. 